http://jmscr.igmpublication.org/home/ ISSN (e)-2347-176x ISSN (p) 2455-0450

crossref DOI: https://dx.doi.org/10.18535/jmscr/v8i3.104

Journal Of Medical Science And Clinical Research

\title{
A randomized controlled trial of ganglion impar block vs ganglion impar block with superior hypogastric plexus block for pain relief in patients of pelvic and perineal cancer
}

\author{
Authors \\ Dr Apoorva Gupta ${ }^{1}$, Dr Sarita Singh², Dr Rajesh Raman ${ }^{3 *}$, Dr Prem Raj Singh , \\ Dr Manish Kumar Singh ${ }^{5}$, Dr Rati Prabha ${ }^{6}$, Dr Shashank Kumar ${ }^{7}$ \\ ${ }^{1}$ Junior resident, Department of Anesthesiology, King George's Medical University, Lucknow \\ ${ }^{2}$ Professor, Professor, Department of Anesthesiology, King George's Medical University, Lucknow \\ ${ }^{3}$ Associate Professor, Department of Anesthesiology, King George's Medical University, Lucknow \\ ${ }^{4}$ Associate Professor, Department of Anesthesiology, King George's Medical University, Lucknow \\ ${ }^{5}$ Assistant Professor, Department of Anesthesiology, King George's Medical University, Lucknow \\ ${ }^{6}$ Assistant Professor, Department of Anesthesiology, King George's Medical University, Lucknow \\ ${ }^{7}$ Senior Resident, Department of Anesthesiology, King George's Medical University, Lucknow \\ *Corresponding Author \\ Dr Rajesh Raman
}

Associate Professor, Department of Anesthesiology, King George's Medical University, Lucknow-226003, Uttar Pradesh, India

\begin{abstract}
We compared the efficacy of Ganglion Impar (GI) block versus Superior Hypogastric Plexus (SHP) with GI block (both combined) for pain relief in patients of pelvic and perineal cancer. This prospective, randomized, double-blinded, controlled study was conducted on 50 patients, 18 to 70 years old with pelvic and perineal cancer pain with baseline VAS score>5.GI neurolysis with 3-5ml 70\% alcohol in one group (group G) and GI along with SHP neurolysis with $10 \mathrm{ml} 70 \%$ alcohol on either side was done in the other group(group S).VAS score, quality of life (QOL) score by Mcgill's quality of life questionnaire, requirement of rescue analgesia post-procedure were assessed. The VAS score decreased in both the groups and was lower in group $S$ than in group $G$, at all intervals except at baseline. QOL also improved in both the groups, the QOL scores being lower in $S$ than $G$ group at all intervals, except at 2 weeks follow up. Rescue analgesic requirement was similar in both the groups except at $2^{\text {nd }}$ and $3^{\text {rd }}$ months when morphine consumption was more in GROUP G. Concluded that GI block and GI with SHP block both provide drastic pain relief in pelvic and perineal cancer pain, pain relief being more when SHP is blocked along with GI. Quality of life is improved, and morphine requirement is reduced in both groups (more in group $S$ ).

Keywords: Ganglion Impar Block, Superior Hypogastric Plexus Block, Pelvic Cancer, Perineal Cancer, Quality of life questionnaire, visual analog scale.
\end{abstract}

\section{Introduction}

$40 \%-50 \%$ of cancer pain can be categorized as exclusively or partly neuropathic. ${ }^{[1-2]}$ Pelvic cancer causes several types of pain, i.e., visceral, neuropathic, and somatic pain. The infiltration of the perineal nerves results in lumbosacral 
plexopathies and complete destruction of the nerve, including perineural lymphatic invasions producing symptomatic sensory loss, causalgia, and deafferentation.

Sympathetic ganglia blocks can be effective in controlling visceral pain. ${ }^{[3]}$ Visceral pain transmitted by sympathetic fibers of pelvic and perineal origin can be treated with ganglionimpar (GIB) in a simple and effective manner. ${ }^{[4]}$ The superior hypogastric plexus (SHP) is a retroperitoneal structure with a predominance of afferent sympathetic nerve fibres. The perception of central pelvic pain is thought mainly to involve transmission through this plexus. Accordingly, blocking or destruction of SHP has long been used to treat pain in the pelvis.

To the best of our knowledge, no study till date has compared the efficacy of ganglion impar block versus a combined ganglion impar with superior hypogastric plexus block for pain relief in patients of pelvic and perineal cancer. Therefore this study was undertaken to compare the two.

\section{Material and Methods}

This prospective randomized double-blinded study was conducted on patients aged 18 to 70 years of pelvic and perineal cancer pain with Visual analog scale (VAS $>5$ ) visiting pain clinic OPD of our institute. Prior institutional ethics committee approval and written informed consent from the patients were obtained. This study was registered with Clinical Trials Registry of India (CTRI/2019/03/018267).Patients giving negative consent, with platelet count $<150,000 / \mathrm{mm} 3$, on anticoagulant therapy, any coagulation disorders or any active local site infection were excluded.

Before the procedure, baseline VAS score was measured and baseline quality of life (QOL) score was measured by filling McGill QOL questionnaire. ${ }^{[5]}$ All the monitors were attached (i.e. pulse oximeter, noninvasive blood pressure, and electrocardiogram). An anesthesia technician, otherwise not involved in the study, prepared the injections (total volume $10 \mathrm{ml}$ ) depending on the group allocated. The groups were:
Group G: Injection of $10 \mathrm{~mL}$ of normal saline followed by $1 \mathrm{~mL}$ of saline was given (for blinding).

Group S: Injection of $10 \mathrm{~mL}$ of $70 \%$ alcohol in saline followed by $1 \mathrm{~mL}$ of saline, on either side was given to avoid the deposition of alcohol within the intervertebral disc material.

The technique used was the same as described by Ahmed et $\mathrm{al}^{[4]}$ in their study. All the parameters including VAS scores were recorded by an assessor who was unaware of the group allocation and did not participate in the neurolysis procedures.Follow-up was done through OPD visits of patients at 1 week and at monthly intervals upto 3 months and through regular telephonic communications regarding pain relief with respect to VAS score, requirement of rescue analgesics and improvement of QOL (using McGill QOL Questionnaire). We also looked for any side effect after the procedure.

Based on previous studies, ${ }^{[1-2]}$ the hypothesized difference of 1.2 in terms of VAS was considered to be clinically significant. Assuming a type I error of 0.05 and a desired power value of $80 \%$ using the two-tailed Z-test for proportions, 16 patients were calculated as the minimum sample size for each group. The significance level of the test was targeted at 0.05 . Considering possible dropouts, 25 patients were included in each group.

\section{Results}

The flow of patients is depicted in Figure 1 according to CONSORT guidelines. There was no difference between groups regarding baseline demographic characteristics (Table1). There was decrease in VAS score from baseline in both the groups, and it was lower in $\mathrm{S}$ group as compared to $G$ group at all intervals. The difference in the VAS score between both the groups was also statistically significant. There was decrease in mean VAS score from baseline in both the groups, and it was lower in steroid group as compared to prolotherapy group at all intervals. The difference in the VAS score between both the groups was 
also statistically significantat all follow up time intervals (Table 2).

There was improvement in quality of life of patients in both the groups from baseline at all the subsequent intervals. At all the time intervals, mean QOL scores were lower in group B as compared to group A, except at 2 weeks. The difference between both the groups was also statistically significant at all the follow up time intervals except at $1^{\text {st }}$ week and $2^{\text {nd }}$ month (Table $3)$.

Total doses of rescue analgesic consumed was more in group G. This further supports the superiority of analgesic efficacy of a combined superior hypogastric plexus and ganglion impar block over a ganglion impar block. (Table 4). The side effect profile of both the groups was almost similar (Table 5).

Table 1 Comparison of demographic variables andbaseline characteristics

\begin{tabular}{|l|c|c|c|}
\hline Characteristic & Group G $(\mathrm{n}=25)$ & Group S (n=25) & p \\
\hline Age(years) & $42.24 \pm 14.58$ & $39.24 \pm 13.55$ & 0.455 \\
\hline Weight $($ Kg) & $60.96+10.95$ & $65.56+9.02$ & 0.112 \\
\hline Height $(\mathrm{cm})$ & $161.68+7.35$ & $165.76+8.25$ & 0.071 \\
\hline Gender & $14(56 \%) / 11(44 \%)$ & $13(52 \%) / 12(48 \%)$ & 1.000 \\
(Male/Female) & & & \\
\hline Diagnosis: & & & 0.332 \\
Caano-rectum & $9(36 \%)$ & $9(36 \%)$ & \\
Ca bladder & $8(32 \%)$ & $3(12 \%)$ & \\
Ca cervix & $0(0 \%)$ & $2(8 \%)$ & \\
Ca endometrium & $2(8 \%)$ & $3(12 \%)$ & \\
Ca ovary & $1(4 \%)$ & $3(12 \%)$ & \\
Ca prostate & $2(8 \%)$ & $1(4 \%)$ & \\
Ca Rectum & $0(0 \%)$ & $2(8 \%)$ & \\
Ca vulva & $3(12 \%)$ & $2(8 \%)$ & \\
\hline
\end{tabular}

Data is presented as Mean \pm SD and number (percentage)

Table 2 Comparison of visual analog scale score at different intervals between the groups

\begin{tabular}{|l|c|c|c|}
\hline VAS & Group G $(\mathbf{n = 2 5})$ & Group $\mathbf{S}(\mathbf{n}=\mathbf{2 5})$ & p-value \\
\hline Baseline & $7.96 \pm 0.61$ & $7.84 \pm 0.80$ & 0.492 \\
\hline 24 hour & $3.52 \pm 1.69$ & $1.56 \pm 1.87$ & $<\mathbf{0 . 0 0 1} *$ \\
\hline 1 week & $2.73 \pm 0.55$ & $1.86 \pm 1.46$ & $<\mathbf{0 . 0 0 1} *$ \\
\hline 2 week & $2.74 \pm 0.56$ & $1.43 \pm 0.51$ & $<\mathbf{0 . 0 0 1 *}$ \\
\hline 1 month & $2.74 \pm 0.45$ & $1.14 \pm 0.73$ & $<\mathbf{0 . 0 0 1 *}$ \\
\hline 2 month & $2.42 \pm 0.51$ & $1.67 \pm 0.73$ & $<\mathbf{0 . 0 0 1} *$ \\
\hline 3 month & $3.00 \pm 0.33$ & $1.43 \pm 0.60$ & $<\mathbf{0 . 0 0 1 *}$ \\
\hline
\end{tabular}

Data is presented as Mean \pm SD, VAS=visual analog scale score, ${ }^{*}=$ statistically significant. $\mathrm{n}$ for group $\mathrm{G}=19 \& \mathrm{~S}=21$ after 24 hours

Table 3 Comparison of Quality of life scores at different intervals between the groups

\begin{tabular}{|l|c|c|c|}
\hline QOL & Group G $(\mathbf{n}=\mathbf{2 5})$ & Group $\mathbf{S}(\mathbf{n}=\mathbf{2 5})$ & p-value \\
\hline Baseline & $77.00 \pm 2.96$ & $77.28 \pm 2.59$ & 0.713 \\
\hline 24 hour & $51.12 \pm 14.06$ & $44.84 \pm 13.02$ & $\mathbf{0 . 0 3 3}$ \\
\hline 1 week & $38.19 \pm 4.31$ & $37.55 \pm 11.28$ & 0.106 \\
\hline 2 week & $34.21 \pm 5.09$ & $39.10 \pm 6.62$ & $\mathbf{0 . 0 1 0}^{*}$ \\
\hline 1 month & $46.21 \pm 4.30$ & $42.38 \pm 2.92$ & $\mathbf{0 . 0 0 4}^{*}$ \\
\hline 2 month & $37.58 \pm 3.42$ & $35.95 \pm 4.04$ & 0.150 \\
\hline 3 month & $41.26 \pm 3.91$ & $38.57 \pm 3.38$ & $\mathbf{0 . 0 2 2}^{*}$ \\
\hline
\end{tabular}

$\mathrm{QOL}=$ Quality of life, Data is presented as Mean \pm SD, *=statistically significant. $\mathrm{n}$ for group $\mathrm{G}=19$ \& $\mathrm{S}=21$ after 24 hours

Table 4 Comparison of daily morphine consumption between the groups

\begin{tabular}{|l|c|c|c|}
\hline Morphine consumption mg/day & Group G $(\mathbf{n}=\mathbf{2 5})$ & Group S $(\mathbf{n}=\mathbf{2 5})$ & p-value \\
\hline Baseline & $145.20 \pm 77.79$ & $154.40 \pm 82.16$ & 0.41 \\
\hline 24 hour & $15.60 \pm 39.80$ & $10.40 \pm 29.51$ & 0.52 \\
\hline 1 week & $0.00 \pm 0.00$ & $0.00 \pm 0.00$ & NA $^{\#}$ \\
\hline 2 week & $3.16 \pm 13.76$ & $0.00 \pm 0.00$ & 0.299 \\
\hline 1 month & $3.16 \pm 13.76$ & $0.00 \pm 0.00$ & 0.299 \\
\hline 2 month & $26.84 \pm 50.89$ & $2.86 \pm 9.02$ & $\mathbf{0 . 0 4 0}^{*}$ \\
\hline 3 month & $44.21 \pm 50.37$ & $18.57 \pm 22.20$ & $\mathbf{0 . 0 4 1}^{*}$ \\
\hline
\end{tabular}


Table 5 Comparison of the two groups with respect to side effect profile

\begin{tabular}{|l|c|c|c|}
\hline Side Effect & Group G (n=25) & Group S (n=25) & p \\
\hline Painon injection & $2(8.0 \%)$ & $1(4.0 \%)$ & 1.000 \\
\hline Requiredrepeat neurolysis & $3(12.0 \%)$ & $2(8.0 \%)$ & 1.000 \\
\hline Transient hypotension & $1(4.0 \%)$ & $0(0.0 \%)$ & 1.000 \\
\hline Transient paresthesia & $2(8.0 \%)$ & $3(12.0 \%)$ & 1.000 \\
\hline
\end{tabular}

Data is presented as Number (percentage)

\section{CONSORT Flow Diagram}

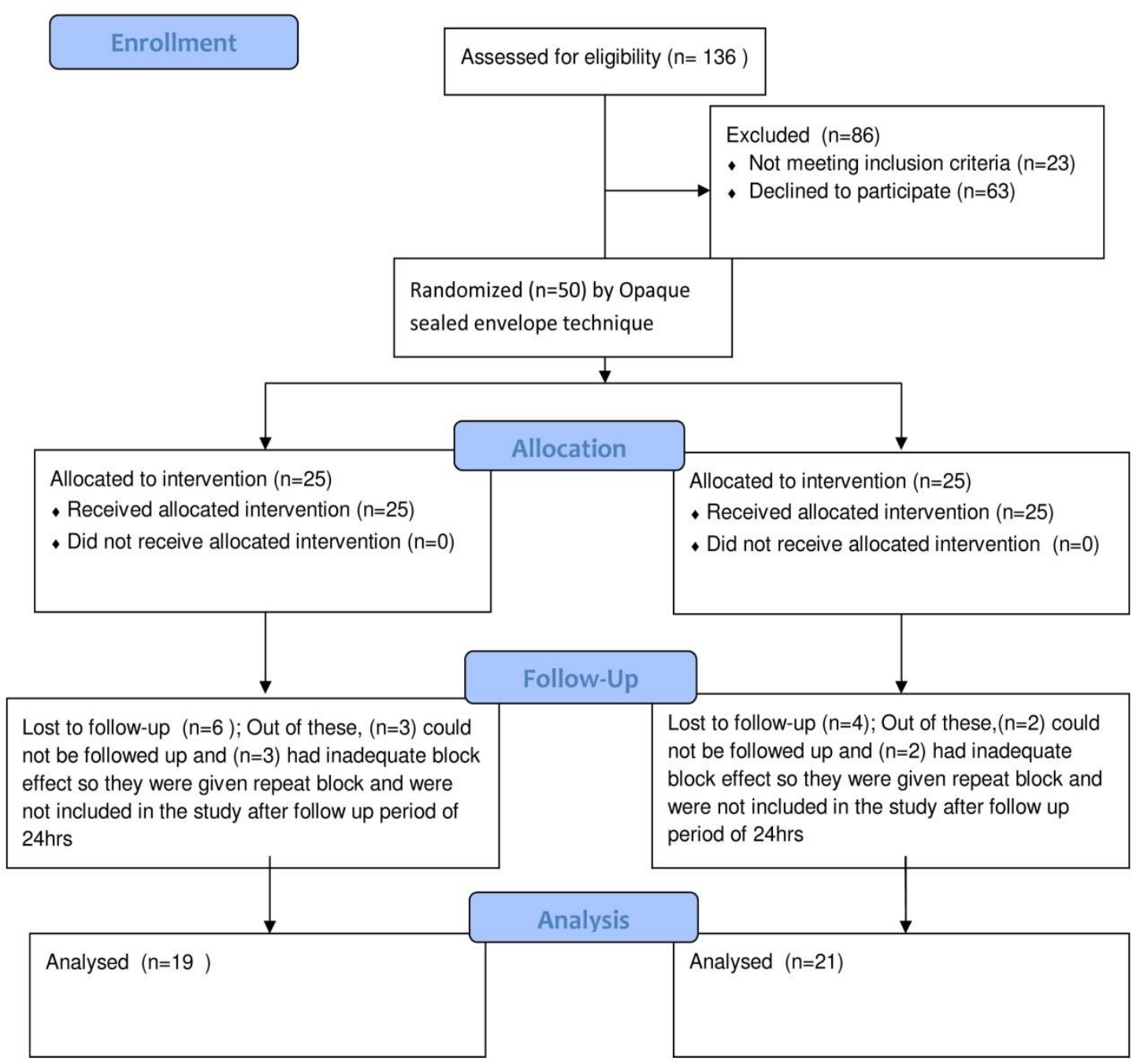

Figure 1.The consort chart.

\section{Discussion}

The present study showed that GIB alone as well as ganglion impar block with SHP block (SHPB) both were effective in the pain management of patients with pelvic and perineal cancer but superior hypogastric plexus block when added on to ganglion impar block was much more effective in relieving pain as well as improving the quality of life of the patients.
Gunduz et al. ${ }^{[6]}$ conducted a case series of neurolytic GIB by trans-coccygeal approach, analyzing its safety and efficacy. In this study, five consecutive patients were given ganglion impar block for chronic pelvic pain using a transcoccygeal approach and were followed up for 2 months. All the patients had clinically significant pain relief with VAS score of 2 till 2months follow up. They described pain relief in 
terms of VAS. Toshniwal et al. ${ }^{[7]}$ conducted study in 16consecutive patients who required ganglion impar block for CPP (chronic pelvic pain) and were followed up for two months. The mean VAS at 2 months in their study was 3.Other studies have also shown improvement in pain scores after GIB..$^{[1,2,8]}$

There are several studies which were done on SHPB alone for alleviating pain of pelvic and perineal cancer. Wechsler et al. ${ }^{[9]}$ performed SHP Block in 7 patients. One procedure resulted in mild pain relief, three in considerable pain relief, one in complete midline pain relief with no change in the lateral pain, and one in complete pain relief. Gamal et $\mathrm{al}^{[10]}$ reported that the transdiscal approach for superior hypogastric plexus block in pelvic cancer pain is easier, safer, and more effective with less side effects than the classic approach. In our study also we have used the transdiscal approach for the superior hypogastric plexus block and it was given with ganglion impar block to see it's add on effect in patients with pelvic and perineal cancer pain. And this group of patients showed better VAS score improvement than in patients in which GI block was given alone.

There are two publications in which GI block and SHP block were given together in pelvic and perineal cancer pain. Ahmed et al. ${ }^{[4]}$ studied the feasibility, safety, and efficacy of combining the block of the SHGP through the postero-median transdiscal approach with the GI block by the trans-sacro-coccygeal approach for relief of pelvic and/or perineal pain caused by pelvic and/or perineal malignancies or any cancer related causes. Fifteen patients who had cancer-related pelvic pain, perineal pain, or both received a combined SHGP neurolytic block through the postero-median transdiscal approach using a 20gauge Chiba needle and injection of $10 \mathrm{~mL}$ of $10 \%$ phenol in saline plus a GI neurolytic block by the trans-sacro-coccygeal approach using a 22gauge $5 \mathrm{~cm}$ needle and injection of 4-6 $\mathrm{mL}$ of $8 \%$ phenol in saline. The VAS score of the patients was $7.87 \pm 1.19$ before the block was performed.
VAS score decreased significantly $(\mathrm{p}=0.001)$ immediately after injection compared with the pre-procedural period and was sustained during all follow-up measurements with maximum reduction by $69.5 \%$ of the baseline VAS score $(\mathrm{P}=0.001)$ observed after one week. Just as in our study, the reduction of VAS from baseline at one week follow up interval was $76.2 \%$. Yeo et $\mathrm{al}^{[11]}$ in their case report mentioned that an Indian lady who suffered from metastatic carcinoma of the cervix experienced tremendous pain and disability despite high doses of narcotics and membrane stabilizers. A ganglion of impar block and a superior hypogastric plexus block were performed with a neurolytic agent. The patient's pain and opioids usage were markedly reduced. Thus, they concluded that neurolytic nerve block can offer a great therapeutic option in selected cancer patients.

The findings in both the above publications are in support of our study, that GI and SHP block are highly efficacious for pain relief in pelvic and perineal cancer. We have compared them (GI \&SHP block) combined together with GI block alone and found that GI with SHP block combined together is more efficacious than GI block alone for pain relief in patients of pelvic and perineal cancer. We could not find any study comparing the above two groups in a single study.

We could not find any study with quality of life by McGill's quality of life questionnaire as primary or secondary outcome variable.

In our study, we compared the improvement in quality of life in terms of score depending on the answers given to McGill Quality of life questionnaire. We observed that though the mean quality of life scores at baseline was comparable in both the groups, the score being slightly higher in group $\mathrm{S}$ as compared to that of group $\mathrm{S}$, but the difference was not statistically significant. At all the subsequent follow up intervals, there was more improvement in the group $S$ except at 2 weeks and the difference in scores between the two groups were statistically significant except at ${ }^{\text {st }}$ week and $2^{\text {nd }}$ month. 
Although, if pain relief was better in $\mathrm{S}$ group than $\mathrm{G}$ group, then theoretically the quality of life should also have been better in $S$ group than $G$ group at all intervals and also statistically significant but QOL has been found to be better in $\mathrm{G}$ group than in $\mathrm{S}$ group at 2 weeks and vice versa at all other intervals, also the difference was found to be insignificant at 1 week and 2 month intervals. This discrepancy can be explained by the fact that most of these patients have been on chemotherapy and radiotherapy due to the debilitating malignancies, which have not been taken into account in our study.

In our study, we also compared requirement for rescue analgesia between group $\mathrm{G}$ and group $\mathrm{S}$ to describe the pain-relieving efficacy of these two groups. We found that there was more requirement of rescue analgesia i.e. morphine tablets post procedure in group $\mathrm{S}$ as compared to that in group $\mathrm{S}$.

As far as the incidence of side effects are concerned, it was similar in both the groups i.e. in $32 \%$ cases of group $\mathrm{G}$ and $24 \%$ cases of $\mathrm{S}$ group. Out of those $32 \%$ cases (8 patients) in group G, 2 had pain on injection, 3 required repeat neurolysis, 1 had transient hypotension and 2 had transient paresthesia. Whereas out of the $24 \%$ cases (6 patients) group S, 1 patient had pain on injection, 2 required a repeat neurolysis and 3 patients had transient paresthesia. There was no significant difference between the two groups with respect to side effects. Our study was not adequately powered to detect difference in side effects, so these findings related to side effects may be validated in studies with larger sample size with adequate power.

\section{Limitations}

One of the limitations of our study was sample size which was not large enough to reveal significant between-group differences for secondary outcome variables. Another limitation was the use of VAS score for assessing pain as it is a subjective test for evaluating outcomes \& is subject to bias. A limited timeframe for the study and ongoing chemotherapy and radiotherapy not being taken into account, which may have a major effect on the quality of life of cancer patients.

\section{Conclusions}

We concluded that both GI block and a combined GI with SHP block are effective in providing pain relief and improving quality of life in patients of pelvic and perineal cancer pain, however a combination of the two is more efficacious than GI block alone in relieving pain as well as improving the quality of life of these patients.

\section{Financial Support and Sponsorship: Nil.}

Conflicts of Interest: There are no conflicts of interest.

\section{Acknowledgement: Nil}

\section{References}

1. Caraceni A, Portenoy RK. An international survey of cancer pain characteristics and syndromes. IASP Task Force on Cancer Pain. Pain 1999; 82: 263-274.

2. Manfredi PL, Gonzales GR, Sady R et al. Neuropathic pain in patients with cancer. $\mathbf{J}$ Palliat Care 2003; 19: 115-118.

3. Haylen BT, de Ridder D, Freeman RM, et al. An International Urogynecological Association (IUGA)/International Continence Society (ICS) joint report on the terminology for female pelvic floor dysfunction. Neurourol Urodyn 2010;29: 4-20.

4. Ahmed DG, MohamedMF, Mohamed SA. Superior hypogastric plexus combined with ganglion impar neurolytic blocks for pelvic and/or perineal cancer pain relief. Pain Physician 2015; 18:E49-E56

5. Rigor BM. Pelvic cancer pain.JSurg Oncol. 2000;75:280-300

6. Gunduz OH, Sencan S, Kenis-Coskun O. Pain relief due to ranssacrococcygeal ganglion impar block in chronic coccygodynia: a pilot study. Pain Med. 2015;16:1278-1281. 
7. Toshniwal GR, Dureja GP, Prashanth SM. Transsacrococcygeal approach to ganglion impar block for management of chronic perineal pain: A prospective observational study. Pain Physician 2007;10:661-6

8. Wootton M. Morphine is not the only analgesic in palliative care: literature review. J Adv Nurs.2004; 45: 527-532.

9. Wechsler RJ, Maurer PM, Halpern EJ, Frank ED. Superior hypogastric plexus block for chronic pelvic pain in the presence of endometriosis: CT techniques and results. Radiology. 1995;196:103-6.

10. Gamal G, Helaly M, Labib YM. Superior hypogastric block: transdiscal versus classic posterior approach in pelvic cancer pain. Clin J Pain. 2006;22:544-547.

11. Yeo SN, Chong JL. A case report on the treatment of intractable anal pain from metastatic carcinoma of the cervix. Annals of the Academy of Medicine, Singapore. 2001;30:632-5. 